\title{
On the Solution of Nonlinear Viscoelastic Material
}

\author{
Hassan Khayyat \\ Faculty of Engineering,Mechanical Engineering Department \\ Shaqra University, Saudi Arabia
}

\begin{abstract}
Here, we consider the problem of twisting of a cylindrical bar of elliptic cross-section. The bar is assumed to consist of a nonlinear viscoelastic material (NVeM). In addition, we consider the bar is subjected to nobody forces and free from forces acting on its lateral surface. Under certain conditions, the problem yields to a nonlinear Volterra integral equation (NVIE) of the second kind. The existence and uniqueness of solution of the integral equation is considered. Moreover, the best numerical method, Product Nystrom method (PNM) is used to obtain the numerical solution. Finally, numerical results are considered and the error estimate is computed
\end{abstract}

Key Words - Nonlinear viscoelastic material, Thermoelasticity- Nonlinear Volterra integral equationProduct Nyström method (PNM). Boundary Value Problem (PVB)

\section{INTRODUCTION}

The theory of Elasticity, thermoelasticity and viscoselastity last few decades, due to its various applications in many fields. The mathematical methods used for solving certain classes of problems of the theory of elasticity, see [1-4].

Viscoelastic mechanics materials have been developing since many decades, such as the method of separation of variables, the use of integral transforms [5] or the theory of functions of a complex variable [6], the variation methods [7] , Galerkin's type methods [8] and the integral equations methods [9,10]. Most of these methods, however, can be effectively used only with problems involving relatively simple geometries to get exact or approximate analytical solutions. To avoid the mathematical difficulties connected with the analytical treatments, the numerical methods can be used, such as the methods based on finite differences or finite elements. The numerical methods have been successful in dealing with problems with complex geometries, but they require tedious calculations and high capacity computing machines in order to discrete the field equations in the bulk and the boundary conditions on the boundary, and to deal with the problems of convergence, stability and computation time saving. An interesting development has occurred by introducing the so-called "Method of Boundary Integral Representation".

The torsion of uniform bars occupies special riches in continuum electrostatic problems because of the simplicity of the stress distribution over the cross-section, reducing it to a simplified two dimensional problem originated by Saint-Venant, see [11-13]. In the work of the linear VeM or NVeM, the problems were formulated as a PVB of the Laplace equation. This can be either a Neumann PVB for the warping function, or Dirichlet PVB for Prandtl's stress function, see [14-21]. Most of the NVeMs lead to a linear or NIEs with continuous or discontinuous kernels. For this, different methods are established to solve the integral equations analytically, see [21-26]. When the analytic methods failed, more attentions is focused in solving the integral equations numerically, see [27-29].

In this paper, we establish the liner and NVIE of the second kind from the boundary value problem of the NVeM. In addition, the existence of a unique solution for NVIE will be discussed and proved. The solution of the linear and 
NVIE will be discussed numerically using PNM. Finally, some numerical results are considered and the error estimate, in each case, is computed.

\section{FORMULATION OF THE NONLINEAR VISCOELASTIC MATERIAL PROBLEM}

Consider the problem of NVeM of twisting cylindrical bar of elliptic cross-section. The bar is subjected to no body forces and free from forces acting on its lateral surface. Assume that the bar has a length $l$ and that one end of it is fixed in the plane $z=0$, with the axis of the bar lying along the positive $z$-axis from $z=0$ to $z=l$. Assume that the major axis of the elliptic cross-section is lying on the $x$-axis from $x=-a$ to $x=a$ and the minor lies on $y$ axis is from $y=-b$ to $y=b$. The bar end in the plane $z=l$ is exhibited to a twisting moment $M$, whose direction along $z$-axis, see Fig (1).

If any point $\mathrm{p}(\mathrm{x}, \mathrm{y}, \mathrm{z})$ in the ellipse cross section goes to $Q(\mathrm{x}+u, y+v, z+w)$, then the deformation $u$ and are given by $\mathrm{u}=\mathrm{x}(\cos \alpha-1)-\mathrm{y} \sin \alpha ; \mathrm{v}=\mathrm{y}(\cos \alpha-1)+\mathrm{x} \sin \alpha$.

Let the angle of twist $\alpha(t)$ be small and variant with time according to the relation $\alpha(t)=\theta(t) z$. Therefore, the components of the displacement vector at any point $p$ take the form

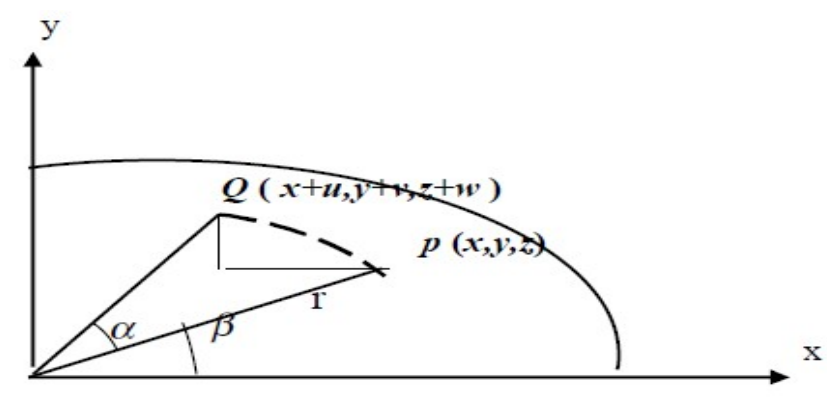

Fig.2

$u=-\theta(t) y z, \quad v=\theta(t) z x, \quad w=\theta(t) \phi(x, y)$.

(1)

Here, $\theta(t)$ is the twist angle per unit length of the bar, $t$ is the time and $\phi(x, y)$ is the torque function called the warping function and

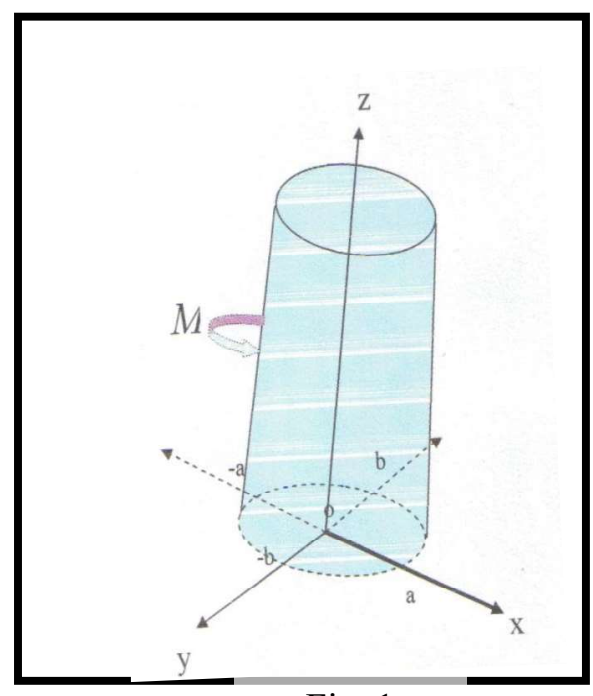

Fig .1 depends only on the $x$ and $y$ coordinates.

Therefore, the strain and stress displacements tensor have, respectively, the forms

$$
\begin{aligned}
& e_{x z}=\frac{\theta(t)}{2}\left(\frac{\partial \phi}{\partial x}-y\right) \quad, \quad e_{y z}=\frac{\theta(t)}{2}\left(\frac{\partial \phi}{\partial y}+x\right) \quad, \quad e_{x x}=e_{y y}=e_{z z}=e_{x y}=0 . \\
& \sigma_{z x}=\mu \theta(t)\left(\frac{\partial \phi}{\partial x}-y\right) \quad, \quad \sigma_{y z}=\mu \theta(t)\left(\frac{\partial \phi}{\partial y}+x\right) .
\end{aligned}
$$

Hence, using the equilibrium equation, we get

$$
\frac{\partial e_{z x}}{\partial y}-\frac{\partial e_{y z}}{\partial x}=-\theta(t) \text {. }
$$




\subsection{FUNDAMENTAL EQUATIONS FOR THE LINEAR AND NONLINEAR VISCOELASTIC MATERIALS}

In the viscoelastic materials, we recall the strain and the stress deviator tensor are defined, respectively in the forms, see [14, 30],

$$
\begin{gathered}
E_{i j}=e_{i j}-\frac{1}{3} e_{k k} \delta_{i j} \quad, \quad e_{k k}=e_{x x}+e_{y y}+e_{z z}, \quad i, j=x, y, z . \\
S_{i j}=\sigma_{i j}-\frac{1}{3} \sigma_{k k} \delta_{i j} \quad, \quad \sigma_{k k}=\sigma_{x x}+\sigma_{y y}+\sigma_{z z}, \quad i, j=x, y, z .
\end{gathered}
$$

In Eqs. (5), (6), $\delta_{i j}$ is the Kronecker $\delta$ function,

The second invariant of strain and stress tensor, respectively take the forms, see [31]

$$
\begin{aligned}
& E^{2}=e_{i j} e_{i j}-\frac{1}{3} e_{k k} e_{k k}, \\
& S^{2}=\sigma_{i j} \sigma_{i j}-\frac{1}{3} \sigma_{k k} \sigma_{k k} .
\end{aligned}
$$

In addition, the principal tensor equations of cubic theory of NVeM are, see [32]

$$
S_{i j}=2 G E_{i j}+\int_{0}^{t} J(t-x) E_{i j} d x+\int_{0}^{t} k(t-x) E^{2} E_{i j} d x .
$$

Here, $G$ is the instantaneous shear modulus of the material, $J(t-x)$ and $k(t-x)$ are the kernel functions for linear and nonlinear relaxation functions of the material, respectively. In the absence of body force, we have $\sigma_{i j}(x, y, z ; t) v_{j}=0 ; \quad i, j=x, y, z$. Here, $v_{j}$ denote the components of the unit vector normal, direction cosine, and $(x, y)$ denote the coordinates of a point of the ellipse $\frac{x^{2}}{a^{2}}+\frac{y^{2}}{b^{2}}=1$, where $a$ is the semi-major axis and $b$ is the semi-minor axis of the ellipse. In addition, the moment of torque at any point is given by $M_{z}(t)=\iint_{R}\left(x \sigma_{y z}-y \sigma_{x z}\right) d x d y$. Where, $\mathrm{R}$ is the cross section of the bar region in the $x y$-plane bounded by the ellipse.

\section{SOLUTION OF THE PROBLEM IN THE FORM OF NVIE}

From the deviator strain of the NVeM, after using Eqs. (5), (7), we obtain

$$
E_{x z}=\frac{\theta(t)}{2}\left(\frac{\partial \varphi}{\partial x}-y\right), \quad E_{y z}=\frac{\theta(t)}{2}\left(\frac{\partial \varphi}{\partial y}+x\right) .
$$

Then, we have

$$
E^{2}(x, y, t)=\frac{\theta^{2}(t)}{4}\left[\left(\frac{\partial \varphi(x, y)}{\partial y}+x\right)^{2}+\left(\frac{\partial \varphi(x, y)}{\partial x}-y\right)^{2}\right] .
$$

In addition, for the stress components, we see that $\sigma_{x z}$ and $\sigma_{y z}$ are the only non-vanishing components of stress, thus we have 


$$
\begin{aligned}
& \sigma_{x z}=2 G E_{x z}+\int_{0}^{t} J(t-x) E_{x z} d x+\int_{0}^{t} k(t-x) E^{2} E_{x z} d x, \\
& \sigma_{y z}=2 G E_{y z}+\int_{0}^{t} J(t-x) E_{y z} d x+\int_{0}^{t} k(t-x) E^{2} E_{y z} d x .
\end{aligned}
$$

By using (3) and (10) in (12) and (13), respectively, we find

$$
\begin{aligned}
\sigma_{z x}(x, y, t)= & G\left(\frac{\partial \phi}{\partial x}-y\right) \theta(t)+\frac{1}{2}\left(\frac{\partial \phi}{\partial x}-y\right) \int_{0}^{t} J(t-x) \theta(x) d x \\
& +\frac{1}{4}\left[\left(\frac{\partial \phi}{\partial x}-y\right)^{3}+\left(\frac{\partial \phi}{\partial y}+x\right)^{2}\left(\frac{\partial \phi}{\partial x}-y\right)\right] \int_{0}^{t} k(t-x) \theta^{3}(x) d x . \\
\sigma_{y z}(x, y, t)= & \left(\frac{\partial \phi}{\partial y}+x\right)\left\{G \theta(t)+\frac{1}{2} \int_{0}^{t} J(t-x) \theta(x) d x\right\} \\
& +\frac{1}{4}\left[\left(\frac{\partial \phi}{\partial x}-y\right)^{2}+\left(\frac{\partial \phi}{\partial y}+x\right)^{2}\right] \int_{0}^{t} k(t-x) \theta^{3}(x) d x .
\end{aligned}
$$

The two formulas (14) and (15) define the stress components of the NVeM. In addition, the momentum reduces to the following form

$$
M(t)=H_{1}\left[G \theta(t)+\frac{1}{2} \int_{0}^{t} J(t-x) \theta(x) d x\right]+\frac{1}{4} H_{2} \int_{0}^{t} k(t-x) \theta^{3}(x) d x,
$$

where

$$
H_{1}=\iint_{R}\left[x \frac{\partial \phi}{\partial y}-y \frac{\partial \phi}{\partial x}+x^{2}+y^{2}\right] d x d y
$$

and

$$
H_{2}=\iint_{R}\left[x \frac{\partial \phi}{\partial y}-y \frac{\partial \phi}{\partial x}+x^{2}+y^{2}\right]\left[\left(\frac{\partial \phi}{\partial y}+x\right)^{2}+\left(\frac{\partial \phi}{\partial x}-y\right)^{2}\right] d x d y .
$$

For the elliptic function, the torque function $\phi(x, y)$ takes the form

$\phi(x, y)=\frac{b^{2}-a^{2}}{a^{2}+b^{2}} x y$

Thus, by calculating $\frac{\partial \phi}{\partial x}$ and $\frac{\partial \phi}{\partial y}$ from Eq. (19), and introducing the results, respectively, in (17) and (18) we get

$$
H_{1}=\frac{\pi a^{3} b^{3}}{a^{2}+b^{2}} . \quad H_{2}=\frac{4 \pi a^{5} b^{5}}{3\left(a^{2}+b^{2}\right)^{2}}
$$

Where, $H_{1}$ is the torsional rigidity and $H_{2}$ is the polar moment of inertia of the cross-section of the bar.

It is known that [32], for the nonlinear elastic materials, the relaxation kernels are given by

$$
J(t-x)=-\lambda(1-v)(t-x)^{-v}, \quad k(t-x)=-K(1-v)(t-x)^{-v} .
$$

Using (21), the formula (16) reduces to the NVIE in the form 


$$
\theta(t)=f(t)+(1-v) \int_{0}^{t}(t-x)^{-v}\left(m \theta(x)+n \theta^{3}(x)\right) d x,
$$

where, we define

$f(t)=\frac{M(t)}{H_{1} G}, \quad m=\frac{\lambda v}{(1-2 v)}, \quad n=\frac{K H_{2}}{4 H_{1} G}, \quad \lambda=\frac{2 G v}{(1-2 v)}, \quad K=\frac{2 G(1+v)}{3(1-2 v)}$.

Here, $\mathrm{K}$ is called the Bulk modulus; $\lambda$ is the Lame's constant, $v(0<v<0.5)$ is the Poison's ratio and $G$ is known as the instantaneous shear modulus of the material.

The formula (22) represents NVIE of the second kind with singular kernel. For a homogeneous elastic material $n=0$; then we have a linear VIE. While for a nonhomogeneous elastic material $\mathrm{m}=0$, we have a NVIE. Many authors have interested in solving the nonlinear integral equations with different kernels using different methods, see [33-38].

\section{NUMERICAL METHODS FOR SOLVING THE NVIE WITH WEAKLY KERNELS}

To discuss the numerical solution of (22), we adapt it, with the aid of (23), in the following form

$\theta(t)=f(t)+\int_{0}^{t}(t-x)^{-v}\left(\lambda_{1} \theta(x)+\lambda_{2} \theta^{3}(x)\right) d x, t \in[0, T] ; T<1$

where $\quad \lambda_{1}=\frac{v(1-v)}{(1-2 v)} ; \quad \lambda_{2}=\frac{a^{2} b^{2}\left(1-v^{2}\right)}{9\left(a^{2}+b^{2}\right)(1-2 v)}$

For using the product Nystrom method, see [27,28], in the space $C[0, T] ; 0 \leq T<1$, we write the kernel of (24) in the form

$k_{\alpha}(|t-x|)=p_{\alpha}(|t-x|) \tilde{k}_{\alpha}(t, x)=(t-x)^{-v} \tilde{k}_{\alpha}(t, x) ; \quad \alpha=1,2 .$,

Where, $p_{\alpha}(|t-x|)$ and $\tilde{k}(t, x)_{\alpha}$ are badly behaved and well-behaved functions of their arguments, respectively.

Then, divide the interval $[0, T]$ in Eq. (24) as the following: $0=t_{0}<t_{1}<t_{2}<\ldots .<t_{N}=T, T<1$. Let

$t=t_{i}=x_{i}=x=i h, i=2,4,6, \ldots, N$ with $h=\frac{T}{N}$, and $N$ is even, then can have

$\theta(i h)-\left(\lambda_{1} \sum_{j=0}^{i} w_{i j} \tilde{k}_{1}(i h, j h) \theta(j h)+\lambda_{2} \sum_{j=0}^{i} w_{i j} \tilde{k}_{2}(i h, j h) \theta^{3}(j h)\right)=f(i h), \theta_{0}=f\left(t_{0}\right)$

Where, the weight functions $w_{i j}$ are given by

$$
\begin{gathered}
w_{i, 0}=\frac{1}{2 h^{2}} \int_{x_{0}}^{x_{2}}\left(x-x_{1}\right)\left(x-x_{2}\right)\left(t_{i}-x\right)^{-v} d x ; \quad w_{i, 2 j+1}=\frac{-1}{2 h^{2}} \int_{x_{2 j}}^{x_{2 j+2}}\left(x-x_{2 j}\right)\left(x-x_{2 j+2}\right)\left(t_{i}-x\right)^{-v} d x \\
w_{i, 2 j}=\frac{1}{2 h^{2}}\left[\int_{x_{2 j}}^{x_{2 j+2}}\left(x-x_{2 j+1}\right)\left(x-x_{2 j+2}\right)\left(t_{i}-x\right)^{-v} d x+\int_{x_{2 j-2}}^{x_{2 j}}\left(x-x_{2 j-2}\right)\left(x-x_{2 j-1}\right)\left(t_{i}-x\right)^{-v} d x\right], \\
w_{i, i}=\frac{1}{2 h^{2}} \int_{x_{i-2}}^{x_{i}}\left(x-x_{i-2}\right)\left(x-x_{i-1}\right)\left(t_{i}-x\right)^{-v} d x ; .
\end{gathered}
$$

The formula (26) represents nonlinear algebraic system, which contains $\left(\frac{N}{2}\right)$ equations. 
In the numerical methods, we need to have some idea of the size of the error involved. Fortunately, the following two definitions enable us to calculate the error of the PNM

Definition 4.1: The estimate local error $R_{i}$ of the numerical methods can be determined by the following equation $R_{i}=\left|\int_{0}^{t}(t-x)^{-v}\left(\lambda_{1} \theta(x)+\lambda_{2} \theta^{3}(x)\right) d x-\left[\lambda_{1} \sum_{j=0}^{i} w_{i j} \tilde{k}_{1}(i h, j h) \theta(j h)+\lambda_{2} \sum_{j=0}^{i} w_{i j} \tilde{k}_{2}(i h, j h) \theta^{3}(j h)\right]\right|(41)$

Definition 4.2: The numerical method is said to be convergent of order $r$, in the interval $[0, \mathrm{~T}]$, if for $N$ sufficiently large, there exist a constant $D>0$ independent of $N$ such that $\left\|\theta(t)-\theta_{N}(t)\right\| \leq D N^{-r}$

\section{APPLICATIONS AND NUMERICAL RESULTS WITH DISCUSSION}

Here, we consider the linear and NVIE (24) of the second kind, when the singular kernel $k(|t-x|)=(t-x)^{-v}$. Then, we use PNM to obtain the numerical solutions of it. For the linear Volterra integral equation, we assume $\lambda_{2}=0$ and for the NVIE we let $\lambda_{1}=0$. In both two cases, we consider $N$ is even finite number and we choose $a=7$ and $b=2.5$ the major and the minor axis of the ellipse, respectively. Also, Poisson ratio $U$ is $0<v<0.5$. Since $0 \leq \mathrm{t} \leq \mathrm{T}<1$ we choose the time $t=0.1,0.5,0.9$, corresponding to the values of $\mathrm{N}=20$. In addition, we consider the exact solution $\theta(t)=t^{2}$.

1) The LVIE can be obtained from Eq. (24) when the axes of the ellipse $a \rightarrow 0$ or $b \rightarrow 0$.

2) In PNM , we take $h=\frac{2 T}{N}, t_{i}=t_{i-1}+h$.

\begin{tabular}{|c|c|c|c|c|c|c|c|c|}
\hline \multirow{3}{*}{$\mathrm{t}$} & \multirow{3}{*}{$\mathrm{N}$} & \multirow[b]{3}{*}{ Exact sol. } & \multicolumn{4}{|c|}{ PNM } & \multicolumn{2}{|l|}{ Linear case } \\
\hline & & & \multicolumn{2}{|c|}{ Polyurethane $\mathrm{v}=0.389$} & \multicolumn{2}{|c|}{ Fibber $v=0.22$} & \multicolumn{2}{|c|}{ Nickel $\quad v=.28$} \\
\hline & & & App. Sol & $\mathrm{E}^{N}$ & App. Sol & $\mathrm{E}^{N}$ & App. Sol & $\mathrm{E}^{N}$ \\
\hline \multirow[b]{4}{*}{0.1} & \multirow[b]{4}{*}{20} & $4.00 \mathrm{E}-04$ & $4.00 \mathrm{E}-04$ & $3.07 \mathrm{E}-9$ & $4.00 \mathrm{E}-04$ & $1.47 \mathrm{E}-11$ & $4.00 \mathrm{E}-04$ & $8.28 \mathrm{E}-11$ \\
\hline & & $3.60 \mathrm{E}-03$ & $3.60 \mathrm{E}-03$ & $6.84 \mathrm{E}-8$ & $3.60 \mathrm{E}-03$ & $3.25 \mathrm{E}-10$ & $3.60 \mathrm{E}-03$ & $1.78 \mathrm{E}-9$ \\
\hline & & $6.40 \mathrm{E}-03$ & $6.40 \mathrm{E}-03$ & $1.55 \mathrm{E}-8$ & $6.40 \mathrm{E}-03$ & $7.26 \mathrm{E}-10$ & $6.40 \mathrm{E}-03$ & $3.95 \mathrm{E}-9$ \\
\hline & & $1.00 \mathrm{E}-02$ & $1.00 \mathrm{E}-02$ & $2.94 \mathrm{E}-8$ & $1.00 \mathrm{E}-02$ & $1.35 \mathrm{E}-9$ & $1.00 \mathrm{E}-02$ & $7.35 \mathrm{E}-9$ \\
\hline \multirow[b]{4}{*}{0.5} & \multirow[b]{4}{*}{20} & $1.00 \mathrm{E}-02$ & $1.00 \mathrm{E}-02$ & $9.86 \mathrm{E}-7$ & $1.00 \mathrm{E}-02$ & 4.73E-9 & $1.00 \mathrm{E}-02$ & $2.30 \mathrm{E}-8$ \\
\hline & & $4.00 \mathrm{E}-02$ & $4.00 \mathrm{E}-02$ & $8.44 \mathrm{E}-6$ & $4.00 \mathrm{E}-02$ & $3.40 \mathrm{E}-8$ & $4.00 \mathrm{E}-02$ & $1.65 \mathrm{E}-7$ \\
\hline & & $1.60 \mathrm{E}-01$ & $1.60 \mathrm{E}-01$ & $9.23 \mathrm{E}-5$ & $1.60 \mathrm{E}-01$ & $2.42 \mathrm{E}-7$ & $1.60 \mathrm{E}-01$ & $1.19 \mathrm{E}-6$ \\
\hline & & $2.50 \mathrm{E}-01$ & $2.50 \mathrm{E}-01$ & $2.23 \mathrm{E}-5$ & $2.50 \mathrm{E}-01$ & $4.58 \mathrm{E}-6$ & $2.50 \mathrm{E}-01$ & $2.26 \mathrm{E}-6$ \\
\hline 0.9 & 20 & $3.24 \mathrm{E}-02$ & $3.24 \mathrm{E}-02$ & $4.46 \mathrm{E}-4$ & $3.24 \mathrm{E}-02$ & $3.97 \mathrm{E}-5$ & $3.24 \mathrm{E}-02$ & $1.88 \mathrm{E}-6$ \\
\hline
\end{tabular}




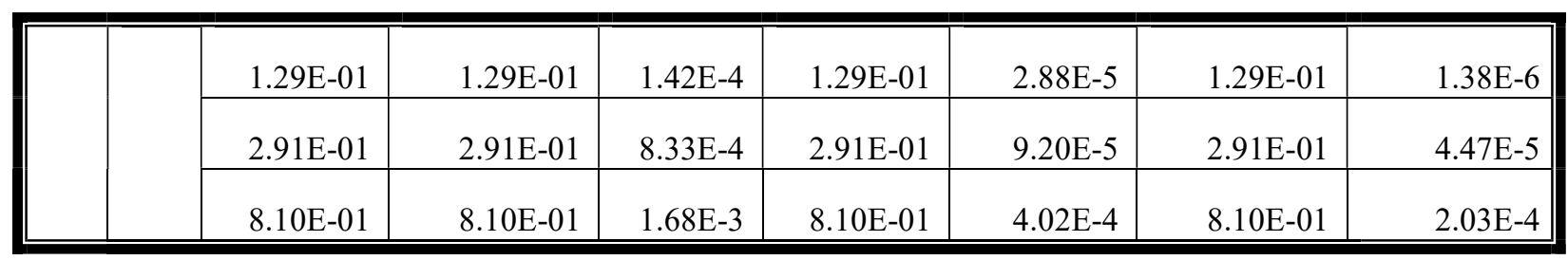

Table (1)

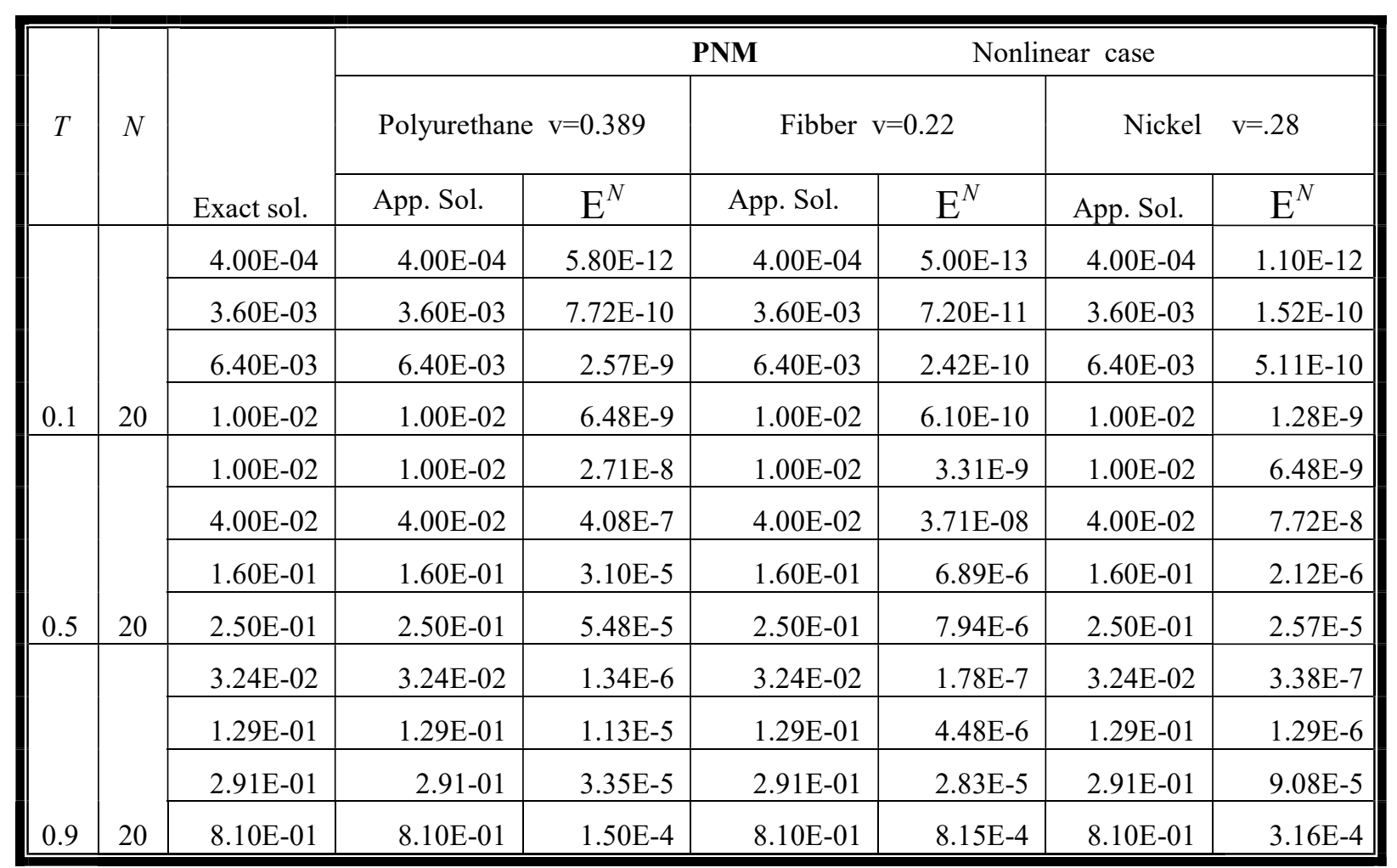

Table (2)

\section{CONCLUSION}

In general, from the previous numerical results of Tables (1)- (2), we deduce the following discussion:

i. For linear and nonlinear cases the error $\mathrm{E}^{N}$ decreases as well as $N$ increases for the materials.

ii. The errors $\mathrm{E}^{N}$ increases as well as the values of time $T$ increasing $(T=0.1,0.5,0.9)$ for linear and nonlinear cases.

iii. If the major $a$ and the minor $b$ axis of the ellipse in the NVIE are increasing, then the $\mathrm{E}^{N}$ are also increasing for each materials.

iv. In the viscoelastic materials, the errors results $\mathrm{E}^{N}$ for linear case are larger than the nonlinear case.

v. We obtain the smallest error $\mathrm{E}^{T}$ for the nonlinear case when $N$ increases and the value of $U$ tend to zero. Under the assumption data at $t=0.2, \quad G=10^{7}, \quad v=0.22$ and, $T=0.1$, we find the smallest errors $\mathrm{E}^{T}$, $\mathrm{E}^{N}$ for $k=3$, are $8.00 \mathrm{E}-15$. 
vi. Due to the $\operatorname{kernel}(t-x)^{-v}$, as the values of $v$ are increasing $(0<v<0.4)$, then the resultant errors $\mathrm{E}^{N}$ are, also increase.

vii. Also, it can be observed from these Tables the following:

1. For Fiber material, $G=10^{7} ; v=0.22$, the minimum value of the errors $\mathrm{E}^{N}$ in linear and nonlinear cases are $8.28 \mathrm{E}-11,5.00 \mathrm{E}-13$. While, the maximum values in the linear case $1.68 \mathrm{E}-3$, and in the nonlinear case is $8.15 \mathrm{E}-4$ at $t$ $=0.9$ for $N=20$.

2. For Nickle material, $G=7900 \times 10^{7}, v=0.28$, the minimum value of the errors in the linear case $1.47 \mathrm{E}-11$, also, the minimum value of the errors in the nonlinear case $5.00 \mathrm{E}-15$, at $T=0.1, t=0.02$ for $N=20$. The maximum values in the linear and nonlinear and 1.68E-3, 3.16E-4, respectively, and each of them occurs at $T=0.9$, for $N=$ 20.

3. For Polyurethane material, $G=0.132 \times 10^{7}, v=0.389$, the minimum value of the errors in the linear case is 3.07E-9. In addition, the minimum value of the errors in the nonlinear case is $5.80 \mathrm{E}-12$. The maximum values in the linear case is $1.68 \mathrm{E}-3$, at $T=0.9, N=20$. While, in the nonlinear is $1.50 \mathrm{E}-4$, occurs at $T=0.9, N=20$.

\section{REFERENCES}

[1] V. V. Zozulya and M. V. Men'shikova, "Dynamic contact problem for a plane with a finite crack," (to be published in Int. Appl. Mech., 38, No. 12 (2002))232-240

[2] VF Chekurin, HV Pokhmurs'ka" mathematical model of cracking of laser-modified metal-powder coatings 2Materials Sciences 40( 5),(2004) 591-596.

[3] V. V. Zozulya, and P. Rivera, "Dynamic fracture mechanics with crack edges contact interaction," in: A. Kassab, M. Chopra, and C. A. Brebbia (eds.), Boundary Elements XX, Computational Mechanics Publications, Southampton, UK and Boston, New York (1998), pp. 2332.

[4] X. Feng, J. Qiu, Y. Ma, J. Lei, Y. Cui, X. Wu, and R.Yang, "Influence of Processing Conditions on Microstructure and Mechanical Properties of Large Thin- Wall Centrifugal Ti-6Al-4V Casting,” J. Mater. Sci.Technol., vol. 32,(2016) pp. 362-371.

[5] Peter Linz, Analytic and Numerical Methods for Volterra Equations, SIM, Philadelphia, 1985.

[6] F. Erdogan, G. D. Gupta and T.S. Cook, Numerical solution of singular integral equations, In: Mechanics of fracture (G. C..Sih, ed), Vol. 1. Methods of analysis and solutions of crack problems. Noordhoff, Leyden. The Netherlands. Chap. 7, 368-425, (1973).

[7] M.A. Abdou, On asymptotic method for Fredholm - Volterra integral equation of the second kind in contact problems, J. Comp. Appl. Math. 154 (2003), 431-446,.

[8] M.A. Abdou, Fredholm - Volterra integral equation with singular kernel, J. Appl. Math. Comp. 137 (2004) pp;231 - 243,.

[9] P. Schiavone, C. Constanda and A. Mioduchowski, Integral Methods in Science and Engineering, Birkhauser Boston, 2002.

[10] R.P. Kanwal, Linear Integral Equations Theory and Technique, Boston, 1996.

[11] J.I. S. Sokolnikoff, Mathematical Theory of Elasticity, five Edition, McGraw-Hill Book Company, New York, 1996.

[12] R. B. Hetnarski, J. Ignaczak, Mathematical Theory of Elasticity, Taylor and Francis, New York, 2004.

[13] N. Noda, R. B. Hetnarski, Y. Tanigawa, Thermal Stresses, Taylor and Francis, New York, 2003.

[14] B. Gross, Mathematical Structure of the Theories of Viscoelasticity, Hemann press, 1983.

[15] Y. C. Fung, Foundations of Solid Mechanics, Prentice - Hall International, 1965.

[16] D. M. Darwish, Stress distribution for nonlinear viscoelastic material under abrupt changes in the state of stress, Presentation at the OSA/TIMS International Meeting, Nashville, Tennessee, USA May12-15, (1991).

[17] E. Treffitz, Mathematical Electrostatic Torsion, Springer, Berlin, fifth Edition, 1981.

[18] N. I. Muskhelishvili, Some Basic Problems of Mathematical Theory of Elasticity, Noordhoff, Holland, 1953.

[19] A. C. Stevenson, Flexure with Shear and Associated Torsion in Prisms of Uni-axial and Symmetric Cross Sections, Cambridge, 1996.

[20] K. G. Nolte, W. N. Findly, A linear compressibility assumption for the multiple integral representation of nonlinear creep of polyurethane, Transactions of ASME's Applied Mech. 441-448, (1970).

[21] W. N. Findly and J. S. Y. Lai, Prediction of uniaxial stress relaxation from creep of nonlinear viscoelastic material, Trans, of Soc. Rheology, 12 (1968) 243-257 
[22] V.M. Aleksandrov, E.V. Covalence, Problems in the Mechanics of Continuous Media with Mixed Boundary Conditions, Nuka Moscow, 1986.

[23] C.D. Green, Integral Equation Methods, Nelson, New York, 1968.

[24] G. Ya. Popov, Contact Problem for a Linearly Deformable Base, Kiev-Odessa, 1982.

[25] F.G. Tricomi, Integral Equations, N. Y. Nelson, 1985

[26] H. Hotchstadt, Integral Equations, N. Y. London, Nelson, 1971

[27] K.E. Atkinson, The Numerical Solution of Integral Equation of the Second Kind, Cambridge, 1997.

[28] L.M. Delves, J.L. Mohamed, Computation Methods for Integral Equations. Philadelphia, New York, 1985.

[29] P. Linz, Analytical and Numerical Methods for Volterra Equations, SIAM, Philadelphia, 1985.

[30] R. T. Shield, Extension and torsion of elastic bars with initial twist, Transactions of ASME, J. Applied Mech., 65 (1982), $779-787$

[31] R. I. Tanner, Engineering Rheology, Oxford University Press, Oxford, 1988.

[32] R. Huilgol, N. Phin Thien, Mechanics of Viscoelasticity, Amsterdam, 1997.

[33] T. Diego, P. Lima, Super convergence of collocation methods for class of weakly singular integral equations, J. Cam. Appl. Math. 218 (2008) 307-316.

[34] G. A. Anastasias, A. Aral, Generalized Picard singular integrals, Compute. Math. Appl. 57 (2009) 821-830

[35] M. A. Abdou, M. M. El-Borai, M. M. El-Kojak, Toeplitz matrix method and nonlinear integral equation of Hammerstein type, J. Com. Appl. Math. 223(2009) 765-776

[36] M. A. Abdou, M. El-Kojak, S. A. Raad, Analytic and numeric solution of linear partial differential equation of fractional order, Global J. and Decision science. Ins. (USA) vol. 13 Issue 3 Version 10 (2013)57-71

[37] L. Thi Phuong Ngoc, Nguyen Thanhlong, On the existence of asymptotically stable solutions for a mixed functional integral equation in N variables, Differential equations and applications, Volume 6, Number 2 (2014), 187-208

[38] E.G. Ladopoulos, Non-linear singular integro-differential equations in Banach spaces by collocation evaluation methods, Univ. J. of Int. Eqs. 1 (2013), 28-38 